\title{
A Case of Pediatric Stroke: Osteosarcoma Embolus in the Internal Carotid Artery
}

\author{
Christopher Troy $^{a}$ Jonathan Sisti ${ }^{a}$ Angel Maldonado-Soto $^{b}$ \\ Gabriella Tosto-D'antonio $^{a} \quad$ Michael L. Miller $^{c}$ Fabrizio Remotti ${ }^{c}$ \\ Grace Mandigo ${ }^{a}$ \\ aDepartment of Neurosurgery, Columbia University Irving Medical Center, New York, NY, USA; \\ bepartment of Neurology, Columbia University Irving Medical Center, New York, NY, USA; \\ 'Department of Pathology and Cell Biology, Columbia University Irving Medical Center, \\ New York, NY, USA
}

\section{Keywords}

Stroke $\cdot$ Pediatric patient $\cdot$ Tumor embolus $\cdot$ Thrombectomy

\begin{abstract}
Stroke in the pediatric population is rare. Despite presentation similar to that seen in the adult patient, the diagnosis in a child can be missed or mistaken for a more common stroke mimic. Due to its rarity, there are no completed pediatric clinical trials investigating best treatment, though guidelines have been extrapolated from adult guidelines and retrospective cohort studies to include some combination of thrombolysis and mechanical thrombectomy. Rarer still is pediatric stroke caused by tumor embolus. We present the case of a young child diagnosed with stroke secondary to osteosarcoma embolism to the left internal carotid artery and review the relevant literature to discuss the considerations and challenges of treatment of stroke in the pediatric population.
\end{abstract}

\section{Introduction}

Pediatric stroke is rare, occurring at rates of $1-2$ per 100,000 children annually [1]. Signs and symptoms are similar to those seen in adults; however, there are often delays in diagnosis due to decreased awareness of pediatric stroke in the community at large. Delays in diagnosis, either through lack of consideration of pediatric stroke or misdiagnosis with a stroke mimic, can lead to worsening ischemic damage and poorer outcomes [1]. In the absence of pediatric clinical trials, treatment guidelines have been extrapolated and adapted from those established 
for the adult population. Tissue plasminogen activator (tPA) has been used in children within the first $4.5 \mathrm{~h}$ from stroke onset, but few supporting data exist, and more robust studies are needed to fully assess its safety and efficacy [2]. Similarly, retrospective studies have shown that thrombectomies improve outcomes in pediatric stroke patients when performed within $6 \mathrm{~h}$ from stroke onset [3-6]. Although more prospective data are required for both tPA and thrombectomy use in children, the current standard of care includes both of these interventions in children presenting with strokes [1]. Herein, we report the exceptionally rare case of a pediatric stroke caused by a tumor embolus in the setting of metastatic osteosarcoma.

\section{Case Report}

The patient was a 6-year-old female with a history of high-grade osteosarcoma with chondroblastic differentiation of the left distal femur status after palliative left leg amputation with hip disarticulation and multiple rounds of chemotherapy, who initially presented with disease progression and new pulmonary metastases. At baseline, she ambulated with a walker due to the amputation, but otherwise had no neurologic deficits. During this admission, she experienced acute right-sided weakness and facial droop. A stroke code was activated, and her exam at that time was significant for a National Institutes of Health Stroke Scale (NIHSS) of 9 for right facial droop with right tongue deviation, right arm and leg strength of $2 / 5$, and decreased sensation on the right. Magnetic resonance imaging and magnetic resonance angiography showed restricted diffusion in the left centrum semiovale extending caudally to the basal ganglia with complete occlusion of the left paraclinoid internal carotid artery (ICA). Large vessel occlusion was called close to the 4.5 -h time mark from her last known normal, and therefore tPA was deferred, and the patient was intubated and taken directly for thrombectomy.

After right femoral access was obtained, an injection of the left ICA confirmed complete occlusion just distal to the takeoff of the ophthalmic artery (shown in Fig. 1a, b). Given the patient's age, small size, and prior amputation, we attempted to work primarily through a 5-French system to avoid femoral artery injury and limb compromise. Aspiration was first attempted with "A Direct Aspiration First Pass Technique" (ADAPT) technique; however, this did not result in any significant recanalization after several attempts. A stent retriever was then deployed across the occlusion; however, again there was no significant recanalization noted. This system was therefore exchanged for a standard 6-French system including aspiration catheter and stent retriever, and clot retrieval was attempted utilizing a variety of thrombectomy techniques. Only trace recanalization was noted, with minimal opacification of the ipsilateral A1 segment (shown in Fig. 1c, d). The procedure was aborted, and after sheath exchange, a 6/7-French Mynx closure device was used. A small specimen was obtained and sent for pathology analysis, which was notable for pleomorphic neoplasm with osteoid and cartilaginous production consistent with high-grade osteosarcoma with osteoblastic and chondroblastic differentiation (shown in Fig. 2). The patient remained in the hospital for almost 2 weeks following the procedure, and at discharge, her exam was notable for persistent dense right-sided hemiparesis and aphasia that require extensive outpatient therapy.

\section{Discussion}

Here, we presented the case of a child who suffered acute ischemic stroke secondary to osteosarcoma embolism to the ICA. Though rare overall, the pediatric cancer patient is at increased risk of stroke given the hypercoagulable state of cancer and the potential for metastases

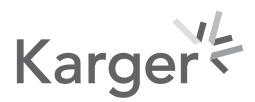



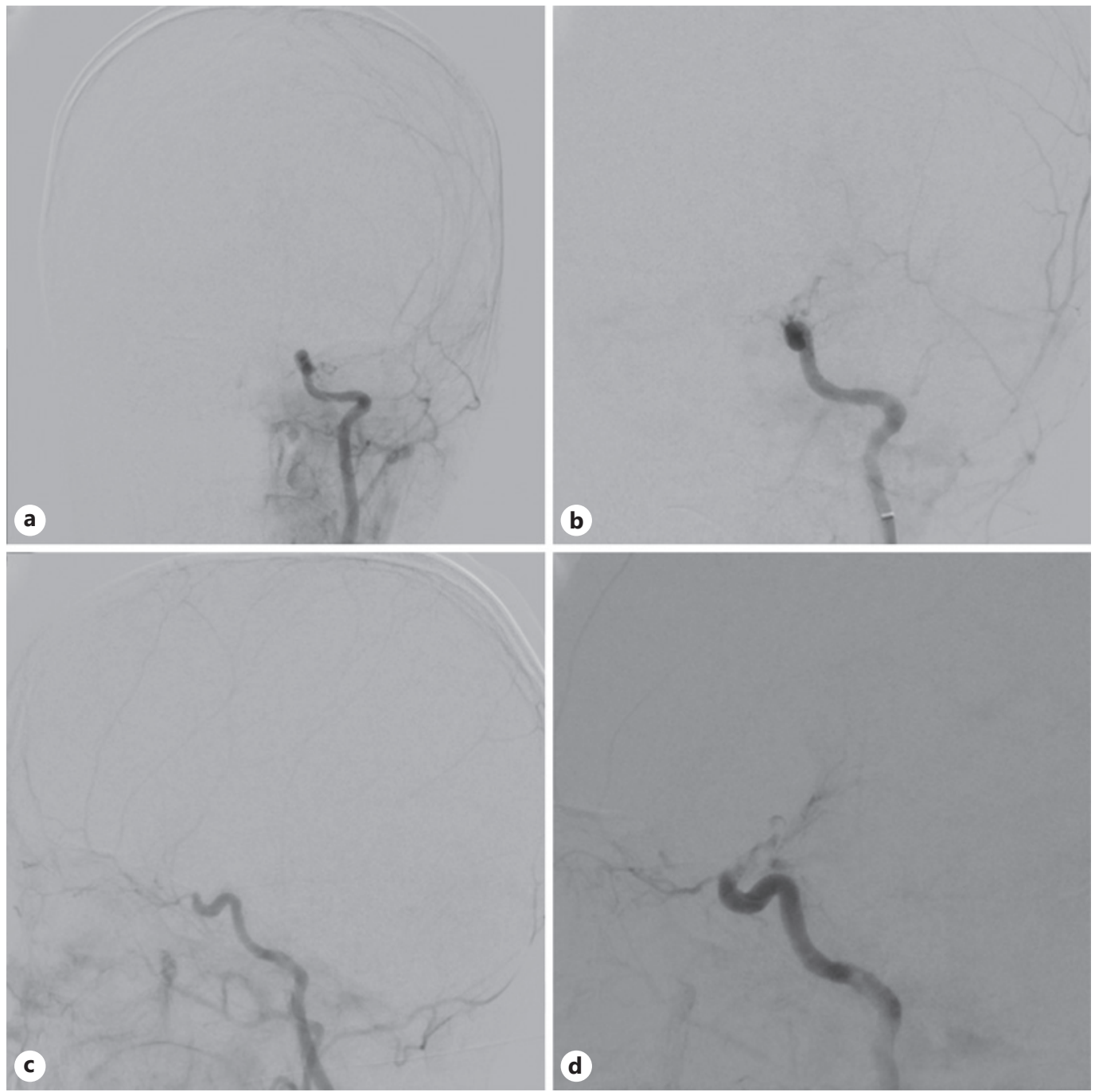

Fig. 1. Angiographic images from before attempted thrombectomy showing complete occlusion of the left ICA distal to the takeoff of the ophthalmic artery (AP (a); lateral (b)). There was no significant recanalization seen after attempted thrombectomy (AP (c); lateral (d)). ICA, internal carotid artery.

and extension or embolism of tumor [7]. Though the source and route of the embolus is not certain, the cause of the occlusion is believed to be tumor embolus versus metastasis or tumor extension for a few reasons. First, embolism is a rare but known complication of metastatic sarcomas [8]. Second, upon partial thrombectomy, we were able to core a sample piece for pathology analysis, which confirmed high-grade osteosarcoma. There was no extravasation of contrast noted after manipulation of the thrombus, meaning the vessel wall was intact and not involved in the embolism. Additionally, the patient has multiple metastatic nodules in her lungs, which can embolize to the brain through a number of routes. Given her large thoracic tumor burden, it is possible that tumor invasion has created an anatomic intrapulmonary shunt between pulmonary veins and arteries. This then leads to a pathologic connection from the right heart into the pulmonary veins, bypassing the smaller pulmonary circulation and allowing tumor to paradoxically embolize from systemic venous circulation to cerebral circulation. An alternative possible source of an embolus could be a cardiac metastasis. A mass was seen in her right ventricle on transthoracic echocardiogram. No mass was seen in her left 

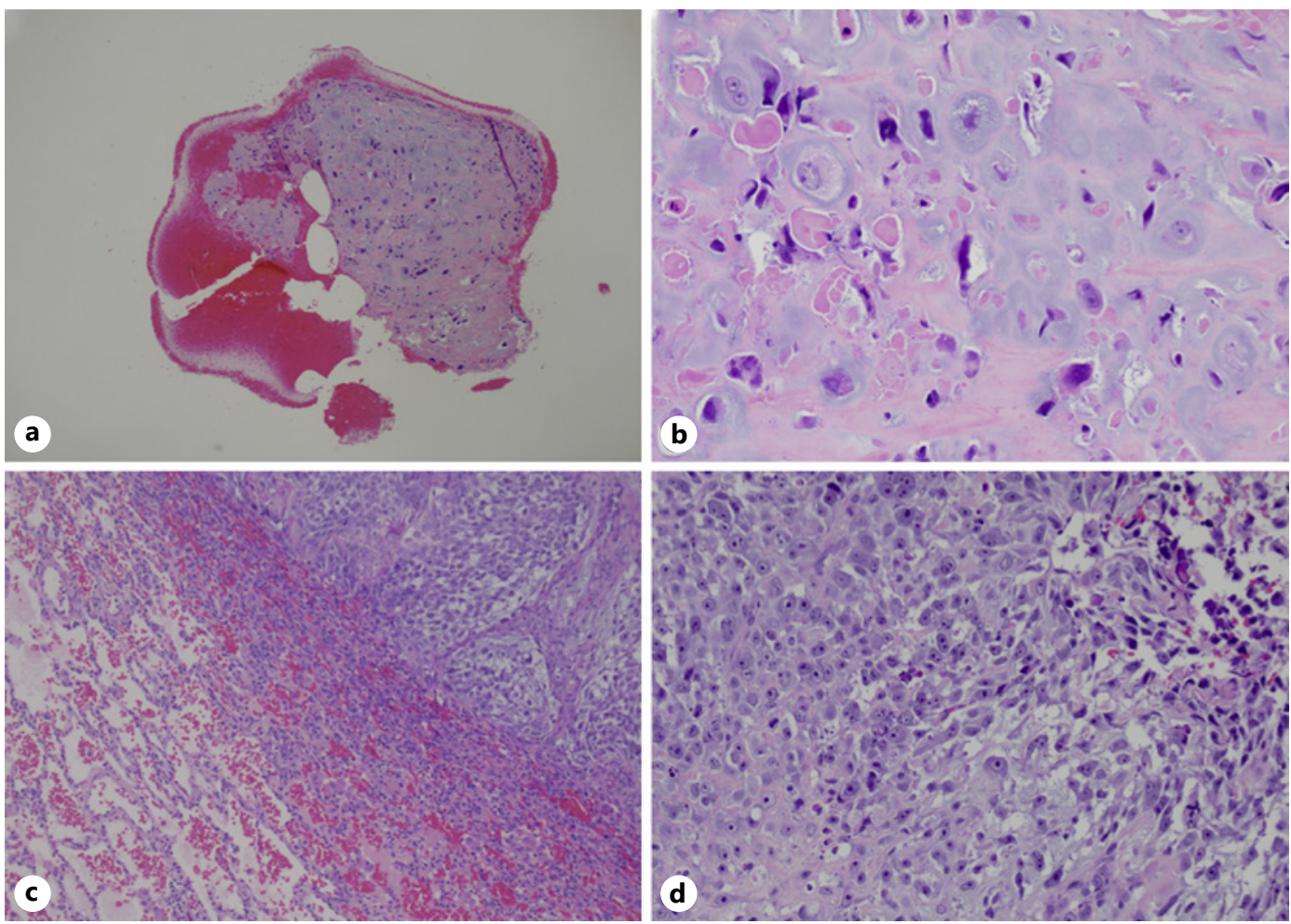

Fig. 2. a, b HE-stained sections of the portion of the embolus extracted from the patient's ICA during attempted thrombectomy showed a pleomorphic sarcoma with osteoblastic and chondroblastic differentiation with osteoid and cartilaginous production consistent with the patient's history of a high-grade osteosarcoma with osteoblastic and chondroblastic differentiation. c, d HE-stained sections from the patient's previous lung biopsy of the right upper lobe lesion was histologically similar, also consistent with high-grade osteosarcoma with osteoblastic and chondroblastic differentiation. ICA, internal carotid artery.

atrium or ventricle, though it remains possible that the embolism could have originated there. Importantly, transthoracic echocardiogram did not show a cardiac or intrapulmonary shunt, and no atrial communication was seen on bubble study. Given these results and her recent pulmonary instrumentation during thoracoscopic biopsy, the more likely possibility is that a small tumor embolized into the pulmonary veins from a pulmonary nodule. This route of embolism is further supported by tumor abutment with pulmonary vessels seen in the left lung on CT of the thorax (shown in Fig. 3). The pattern of enhancement of the inferior pulmonary tumor shows draining into the adjacent pulmonary vein as shown in Figure $3 \mathrm{~b}$, which suggests a possible route of embolism.

There have been few case reports of tumor embolus from osteosarcoma causing stroke. Involvement of the skull and brain [9] and involvement of major neck vessels has been documented, but all of these reports note extravascular compression of vessels versus vascular infiltration and occlusion by tumor [10]. To our knowledge, no such report exists of similar involvement of intracranial vessels causing stroke. Of the reports pertaining to stroke in the sarcoma patient, all describe some sort of cardiopulmonary manifestation of disease, consistent with the presentation of the patient included herein $[11,12]$. Importantly, none involve children, making this case report the first of its kind.

Only 3 cases of endovascular thrombectomy for noncardiac tumor embolus to major cerebral vessels have been reported. In one, Soto et al. [13] report the history of a 57-year-old woman with known chondroblastic osteosarcoma of the chest wall with renal metastases and 
Fig. 3. CT of the thorax from 2 weeks prior to stroke presentation showing tumor abutment with left superior pulmonary artery (a) and left inferior pulmonary vein (b).
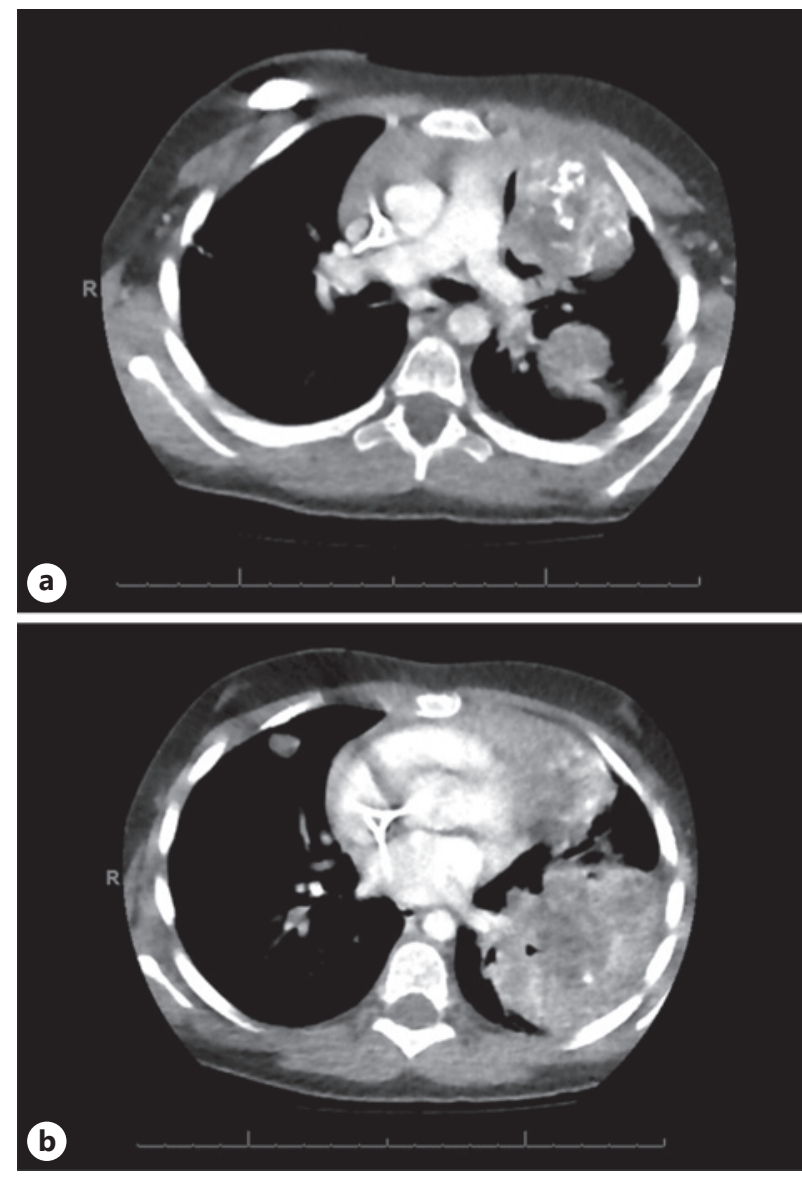

deep vein thrombosis who presented with acute right-sided weakness and aphasia. She was noted to have large masses in both atria which were thought to be the nidus for tumor embolus. While this case and others $[14,15]$ demonstrate successful endovascular mechanical thrombectomy for tumor emboli in adults, mechanical thrombectomy in the pediatric population presents additional technical challenges given the smaller size of the patients and their vasculature, which can significantly limit device selection. In one of the larger retrospective studies of thrombectomies in children, Sporn et al. [6] showed that younger children had poorer outcomes in their group, which could be partially due to the smaller vessel size and more difficult access in those patients.

\section{Conclusion}

We present the rare case of pediatric stroke secondary to osteosarcoma embolus. To our knowledge, this has not been previously reported. Delays in recognition and treatment of pediatric stroke can lead to devastating outcomes. Stroke should remain on the differential diagnosis for altered mental status in children, especially with a history of intrapulmonary neoplasm. If stroke in the pediatric patient is confirmed, mechanical thrombectomy should be attempted when tumor embolization is the suspected mechanism of occlusion. Though successful cases of recanalization in the setting of tumor embolism have been previously reported, this case highlights the technical challenges of mechanical thrombectomy for tumor embolus of this histopathology in the pediatric patient. 


\section{Statement of Ethics}

Written informed consent was obtained from the patient's parent for publication of this case report and any accompanying images.

\section{Conflict of Interest Statement}

The authors have no conflicts of interest to declare.

\section{Funding Sources}

There are no sources of funding to report.

\section{Author Contributions}

Christopher Troy assisted in the conception and discussion of the report with the other authors. He was the primary author of the report and helped throughout the editing process. Jonathan Sisti assisted in the drafting and editing of the report. As the endovascular neurosurgery fellow in charge of her care, Dr. Sisti provided valuable insight into the treatment choices and challenges seen during thrombectomy. Angel Maldonado-Soto assisted in the drafting and editing of the report. As the neurology resident responsible for the patient's pre- and postoperative care, Dr. Maldonado-Soto contributed unique discussion concerning the approach to and treatment options for stroke in the pediatric patient. Gabriella TostoD'Antonio assisted with the discussion of the report. She was an integral member of the group for drafting and editing this final draft for submission. Michael Miller contributed to the histopathologic correlation, case discussion, and writing of the draft. He assisted in the editing of the report and together with Dr. Remotti provided the pathology figures and discussion. Fabrizio Remotti contributed to the histopathologic correlation, case discussion, and writing of the draft. As an expert in sarcoma, Dr. Remotti was responsible for officially confirming the diagnosis of osteosarcoma. He assisted in the drafting and editing of the report and together with Dr. Miller provided the pathology figures and discussion. He also aided in the discussion and conception of the report, especially concerning the rare occurrence of sarcoma causing stroke. Grace Mandigo was the attending physician in the care of the patient. Through her experience as an endovascular neurosurgeon, Dr. Mandigo assisted in discussion and editing of this report. She confirmed the uniqueness of the case and validity of the discussion. All authors have given final approval of this version to be published. All authors agree to be accountable for all aspects of the work and ensure that questions related to the accuracy or integrity of any part of the work are appropriately investigated and resolved.

\section{References}

1 Ferriero DM, Fullerton HJ, Bernard TJ, Billinghurst L, Daniels SR, DeBaun MR, et al. Management of stroke in neonates and children: a scientific statement from the American Heart Association/American Stroke Association. Stroke. 2019 Mar;50(3):e51-96.

2 Amlie-Lefond C, Shaw DWW, Cooper A, Wainwright MS, Kirton A, Felling RJ, et al. Risk of intracranial hemorrhage following intravenous tPA (tissue-type plasminogen activator) for acute stroke is low in children. Stroke. 2020 Feb;51(2):542-8.

\section{Karger's}


3 Goeggel Simonetti B, Cavelti A, Arnold M, Bigi S, Regényi M, Mattle HP, et al. Long-term outcome after arterial ischemic stroke in children and young adults. Neurology. 2015 May 12;84(19):1941-7.

4 Bigi S, Dulcey A, Gralla J, Bernasconi C, Melliger A, Datta AN, et al. Feasibility, safety, and outcome of recanalization treatment in childhood stroke. Ann Neurol. 2018 Jun;83(6):1125-32.

5 Cappellari M, Moretto G, Grazioli A, Ricciardi GK, Bovi P, Ciceri EFM. Primary versus secondary mechanical thrombectomy for anterior circulation stroke in children: an update. J Neuroradiol. 2018 Mar;45(2):102-7.

6 Sporns PB, Sträter R, Minnerup J, Wiendl H, Hanning U, Chapot R, et al. Feasibility, safety, and outcome of endovascular recanalization in childhood stroke: the save childs study. JAMA Neurol. 2020 Jan 1;77(1):25-34.

7 Rogers LR. Cerebrovascular complications in patients with cancer. Semin Neurol. 2004 Dec;24(4):453-60.

8 Latchana N, Daniel VC, Gould RW, Pollock RE. Pulmonary tumor embolism secondary to soft tissue and bone sarcomas: a case report and literature review. World J Surg Oncol. 2017 Aug 30;15(1):168.

9 Weil RJ, Lonser RR, Quezado MM. CNS manifestations of malignancies: case 2. Skull and brain metastasis from tibial osteosarcoma. J Clin Oncol. 2005 Jun 20;23(18):4226-9.

10 Kallala R, Nikkhah D, Nix P, Woodhead C. Primary extraskeletal Ewing sarcoma involving the carotid artery: a case report and review of the current literature. Ann R Coll Surg Engl. 2012 May;94(4):e141-3.

11 Kornfeld SVA, Saper C, Geltman E, Bobzien B, Smith M. Neurologic deterioration and death in a 27-year-old man with chondrosarcoma. Am J Med. 1983 Apr;74(4):663-71.

12 Limper AH, Prakash UB, Kokmen E, Callahan MJ. Cardiopulmonary metastatic lesions of osteosarcoma and associated cerebral infarction. Mayo Clin Proc. 1988 Jun;63(6):592-5.

13 Soto JM, Reed LK, Benardete EA. Successful endovascular removal of a chondroblastic osteosarcoma embolus within the left middle cerebral artery. Proc (Bayl Univ Med Cent). 2020 Jul;33(3):451-2.

14 Zander T, Maynar J, López-Zárraga F, Herrera R, Timiraos-Fernández JJ, Saraceni A, et al. Mechanical thrombectomy in patients with tumour-related ischaemic stroke. Interv Neuroradiol. 2016 Dec;22(6):705-8.

15 Pop R, Mihoc D, Manisor M, Richter JS, Lindner V, Janssen-Langenstein R, et al. Mechanical thrombectomy for repeated cerebral tumor embolism from a thoracic sarcomatoid carcinoma. J Neurointerv Surg. 2018 Oct; 10(10):e26. 